\title{
Rumor Spreading and Degree-Related Preference Mechanism on a Small-World Network
}

\author{
Zhi Zhu, Fengjian Liu, Dongsheng Liao \\ Humanities and Social Sciences School, National University of Defense Technology, Changsha, China \\ Email: kdbybs@126.com
}

Received July $3^{\text {rd }}, 2012$; revised August $6^{\text {th }}, 2012$; accepted August $19^{\text {th }}, 2012$

\begin{abstract}
An alternate model for rumor spreading over small-world networks is suggested, of which two rumors (termed rumor 1 and rumor 2) have different nodes and probabilities of acceptance. The propagation is not symmetric in the sense that when deciding which rumor to adopt, high-degree nodes always consider rumor 1 first, and low-degree nodes always consider rumor 2 first. The model is a natural generalization of the well-known epidemic SIS model and reduces to it when some of the parameters of this model are zero. We find that rumor 1 (preferred by high-degree nodes) is dominant in the network when the degree of nodes is high enough and/or when the network contains large clustered groups of nodes, expelling rumor 2. However, numerical simulations on synthetic networks show that it is possible for rumor 2 to occupy a nonzero fraction of the nodes in many cases as well. Specifically, in the NW small-world model a moderate level of clustering supports its adoption, while increasing randomness reduces it.
\end{abstract}

Keywords: Rumor; Degree-Related Preference Mechanism; Small-World Network

\section{Introduction}

Rumor spreading is a complex socio-psychological process. Pioneering contributions to their modeling, based on epidemiological models, date back to (Landahl, 1953; Rapoport, 1952). In those days both deterministic models and stochastic models were used, and the former were so simple that they were solved analytically and regarded as the first approximation of the latter. To above study, Daley and Kendall (Daley, 1965) explained the importance of dealing with stochastic rumor models rather than deterministic ones, henceforth stochastic models have been actively studied (Cane, 1966; Sudbur, 1985; Watson, 1987). The basic rumor spreading model which they used is called Daley-Kendall model after Daley (Daley, 1965).

The most popular model for information or rumor spreading, introduced by Daley and Kendall (Daley, 1964) is conceptually similar to the SIR model for epidemiology. Other widely used models for describing collective social behavior are the threshold models, first proposed by Granovetter in (Granovetter, 1978). Each individual has a specific threshold, based on which a binary decision is made. More formal definitions which take social network structure into account have appeared since, the simplest version of which is the linear threshold model (Kempe, 2003). A variant of the threshold model has been used, for example, in (Guardiola, 2002) for describing diffusion of innovations in a population, and the effects of network topology have been analyzed by Yunhao Liu (Liu, 2011).

However, the co-hypothesis of above research is that only one type of rumor spreads through networks. In this paper we propose a model of rumor spreading, where two different types of rumor affect the nodes, and consider the behavior of the model on a small-world network.

\footnotetext{
${ }^{*}$ This work was supported in part by the National Natural Science Foundation of China under Grant 91024030 (Modeling crowd psychology and behaviors based on multi-agent theory for commonality secure).
}

Our model follows this idea of the SIS epidemiological model. Nodes are divided into three classes: ignorants, rumor 1 spreaders, and rumor 2 spreaders. Namely, each node is "susceptible" to the effects of two kinds of "infections" and is able to recover from them as well. Moreover, the source of rumor 1 and rumor 2 both have special meaning, and some nodes will always consider one of them first. This formulation could be significant for describing four $\mathrm{w}-\mathrm{o}-\mathrm{m}$ processes circulating in a social network. For example, when two opposing information appear at the same time, both of them have a special meaning in the public eye. But high degree means the one can get information more, easier and more quickly (Christley, 2005), to get the reality and choose which information to believe.

Finally, we outline a study which is close to our work in the sense that two rumor types spread through the network. Namely, in (Goldenberg, 2007), Goldenberg investigated the effects of both positive and negative w-o-m on a firm's profits. In this study, negative w-o-m is limited to traveling two hops away from its source. Trpevski (Trpevski, 2010) made an advance to investigate both rumor types with different probabilities of acceptance. In this study, rumor 1 is limited to be considered first. In our model, both rumors can be selected by different types of nodes.

We structure the paper as follows. After defining the model fully, we analyze the stability of its dynamics in Rumor Spreading Model. In Behavior of the Model on Small-World Network we describe the behavior of the model on NW small-world topology and confirm, via further analysis and dissemination simulations, a number of our calculations. We offer some concluding remarks and points out potential research directions in Conclusions and Discussion.

\section{Rumor Spreading Model}

\section{Definition of the Model}

Consider a clustered populations composed by $\mathrm{N}$ individuals, 
connected by a interaction structure which is represented by graph $G=(V, E)$, with $V$ and $E$ denote the node and the edges, respectively. $A$ denotes the adjacency matrix of the graph $G$, and $a_{i j}=1$ if $(i, j) \in E$ and $a_{i j}=0$ otherwise. Our model is a discrete stochastic model, describing rumor spreading in a small-world network. There are two different types of rumor (rumor 1 and rumor 2) spreading from two different sources. At time $k$, each node $I$ only adopts one of three possible states: 1,2 , and 3 . The state of the node is indicated by a status vector,

$$
s_{i}(k)=\left[\begin{array}{lll}
s_{i}^{1}(k) & s_{i}^{2}(k) & s_{i}^{3}(k)
\end{array}\right]^{T}, i \in 1, \cdots, N .
$$

State 1 or 2 signifies that there is a adopter or spreader of rumor 1 or 2 . A node being in state 3 indicates it is a neutral in relation to the two rumors. Every status vector above is comprised by two sub-states, which denote the degree of nodes. Suppose $s_{i}^{j 1}(k)$ or $s_{i}^{j 2}(k)(j=1,2,3)$ be the status vector of node $i$, signifying it is a high-degree or low-degree node in state $j$. The state of node $i$ becomes

$$
\begin{gathered}
s_{i}^{1}(k)=\left[s_{i}^{11}(k), s_{i}^{12}(k)\right], \\
s_{i}^{2}(k)=\left[s_{i}^{21}(k), s_{i}^{22}(k)\right], \\
s_{i}^{3}(k)=\left[s_{i}^{31}(k), s_{i}^{32}(k)\right], \\
\text { i.e., } s_{i}^{j}(k)=\left[s_{i}^{j 1}(k), s_{i}^{j 2}(k)\right] \quad(j=1,2,3) . \\
\theta^{31}(k)=s_{i}^{31}(k) / s_{i}^{3}(k), \\
\theta^{32}(k)=s_{i}^{32}(k) / s_{i}^{3}(k)=1-\theta^{32}(k)
\end{gathered}
$$

Let

be the fraction of nodes with high or low degree in state 3 , with

$$
s_{i}^{31}(k)=\sum_{i=1}^{N} d_{i}
$$

$d_{i}=1$ if degree $_{i} \geq m_{0}$, otherwise, $d_{i}=0$. The critical degree $m_{0}$, distinguishing the high-degree nodes from low-degree nodes, is defined according to special situation. In a given network, both of the fractions are calculable though simulation. In order to facilitate the mathematical analysis, we rewrite $\theta^{31}(k)$ and $\theta^{32}(k)$ as $\theta(k)$ and $1-\theta(k)$.

Suppose the probability mass function of node $i$ is

$$
p_{i}(k)=\left[p_{i}^{1}(k), p_{i}^{2}(k), p_{i}^{3}(k)\right]
$$

at time $k$. For every node $i$ it states the probability of being in each potential state at time $k$. Then the dynamics equations of each node, i.e., the evolution of the model can be defined as

$$
\begin{gathered}
p_{i}^{1}(k+1)=s_{i}^{31}(k) f_{i}+s_{i}^{32}(k)\left(1-g_{i}\right) f_{i}+a_{1} s_{i}^{1}(k) \\
p_{i}^{2}(k+1)=s_{i}^{31}(k)\left(1-f_{i}\right) g_{i}+s_{i}^{32}(k) g_{i}+a_{2} s_{i}^{2}(k) \\
p_{i}^{3}(k+1) \\
=s_{i}^{31}(k)\left(1-f_{i}\right)\left(1-g_{i}\right)+s_{i}^{32}(k)\left(1-g_{i}\right)\left(1-f_{i}\right) \\
+\left(1-a_{1}\right) s_{i}^{1}(k)+\left(1-a_{2}\right) s_{i}^{2}(k) \\
=s_{i}^{3}(k)\left(1-f_{i}\right)\left(1-g_{i}\right)+\left(1-a_{1}\right) s_{i}^{1}(k)+\left(1-a_{2}\right) s_{i}^{2}(k) \\
\quad s_{i}^{T}(k+1)=\text { MultiRealize }\left[p_{i}^{T}(k+1)\right]
\end{gathered}
$$

So, Equation (1) could be

$$
\begin{gathered}
p_{i}^{1}(k+1)=\theta(k) s_{i}^{3}(k) f_{i}+[1-\theta(k)] s_{i}^{3}(k)\left(1-g_{i}\right) f_{i} \\
+a_{1} s_{i}^{1}(k), \\
p_{i}^{2}(k+1)=\theta(k) s_{i}^{3}(k)\left(1-f_{i}\right) g_{i}+[1-\theta(k)] s_{i}^{3}(k) g_{i}+a_{2} s_{i}^{2}(k) \\
p_{i}^{3}(k+1) \\
=\theta(k) s_{i}^{3}(k)\left(1-f_{i}\right)\left(1-g_{i}\right)+(1-\theta(k)) s_{i}^{3}(k)\left(1-g_{i}\right) \\
\left(1-f_{i}\right)+\left(1-a_{1}\right) s_{i}^{1}(k)+\left(1-a_{2}\right) s_{i}^{2}(k) \\
=s_{i}^{3}(k)\left(1-f_{i}\right)\left(1-g_{i}\right)+\left(1-a_{1}\right) s_{i}^{1}(k)+\left(1-a_{2}\right) s_{i}^{2}(k) \\
s_{i}^{J}(k+1)=\text { MultiRealize }\left[p_{i}^{J}(k+1)\right] .
\end{gathered}
$$

Here, MultiRealize['] performs the realization for probability distribution given with $p_{i}^{J}(k+1), a_{1}$ and $a_{2}$ are parameters $\left(a_{1}, a_{2} \in[0,1]\right)$. We define $f_{i}$ and $g_{i}$ as

$$
f_{i}(k)=1-\prod_{j=1}^{N}\left[1-\beta \alpha_{i j} s_{j}^{1}(k)\right], g_{i}(k)=1-\prod_{j=1}^{N}\left[1-\gamma \alpha_{i j} s_{j}^{2}(k)\right] \text {. }
$$

In Equation (3), $\beta$ and $\gamma$ are parameters $(\beta, \gamma \in[0,1])$. If $a_{2}=0, \gamma$ $=0, \theta(k)=1$ and no node in status 2 initially, a discrete stochastic SIS model can be obtained as a special case of our model. Then Status 1 and status 3 is the infective or susceptible state respectively, with the curing rate being $\delta=1-a_{1}$ (Trpevski, 2010).

The degree-related preference mechanism of spreading is the following. All nodes in status 1 try to spread rumor 1 to all of their neighbors at time $k$, with probability $\beta$ and the transmittals are independent of other. All nodes in status 2 also send rumor 2 to all of their neighbors with probability $\gamma$. Therefore, $f_{i}$ and $g_{i}$ are the probabilities that node $i$ receives rumor 1 or rumor 2 from its neighbor supporting rumors 1 or 2, accordingly. However, note from the formulation of Equation (2) that node $i$ with high degree will become an adopter of rumor 2 at $(k+1)$ only if, at time $k$, it does not receive a rumor 1 from its neighbors, or node $i$ with low degree will become an adopter of rumor 1 at $(k$ +1 ) only if, it does not receive rumor 2 . More precisely, the probability of high-degree nodes converting from an undecided status 3 to status 1 is not $f_{i}$, but multiplied by $\left(1-g_{i}\right)$ which in general is smaller than 1 . Conversely, node $i$ with high degree can become an adopter of rumor 1 regardless of whether it receives rumor 2 or not. It is the same to nodes with low-degree nodes. In this way, our model has performed two preferences in each node for the rumor 1 and 2. Additionally, after supporting a particular kind of rumor, the nodes in state 1 or 2 continue to reserve their status with a rate of $a_{1}$ or $a_{2}$, respectively, i.e., they convert back to status 3 at a rate of $\left(1-a_{1}\right)$ or $\left(1-a_{2}\right)$. Hence, parameters $a_{1}$ and $a_{2}$ signify the remembrance rate of each rumor, or how long nodes want to support the adopted rumor before abandoning it and converting back to undecided status (see Figure 1).

Our model is potentially suitable for a number of real-world situations. In a structured organization, when a grave even happened, managers with high degree, having more number of contacts with different people and shorter path between other individuals, can get more information about the reality and adopt a kind of information. However, employees with low degree, can not get the first hand information, and would like to support an opposing rumor. In marketing circumstances, one 
can imagine two products competing for the market share with similar customer orientation. Therefore, it is safe to use our model to simulate the spread of word-of-mouth about two opposing information from different spreader, or two different products.

Suppose the total number of nodes in statuses 1, 2, and 3 at time $k$, be

$$
\begin{aligned}
& X(k)=\sum_{i=1}^{N} s_{i}^{1}(k), \\
& Y(k)=\sum_{i=1}^{N} s_{i}^{2}(k)
\end{aligned}
$$

and

$$
Z(k)=\sum_{i=1}^{N} s_{i}^{3}(k)=Z_{1}(k)+Z_{2}(k)
$$

respectively. Suppose the number of high degree and low degree nodes in stratus 3 be

$$
\begin{gathered}
Z_{j 1}(k)=\sum_{i=1}^{N} s_{i}^{j 1}(k), \\
Z_{j 2}(k)=\sum_{i=1}^{N} s_{i}^{j 2}(k),(j=1,2,3),
\end{gathered}
$$

respectively. Suppose $N_{1}=E[X(\infty)], N_{2}=E[Y(\infty)]$, and

$$
\begin{gathered}
N_{3}=E[Z(\infty)]=N_{31}+N_{32}, \\
N_{j 1}=E\left[Z_{j 1}(\infty)\right], \\
N_{j 2}=E\left[Z_{j 2}(\infty)\right],(j=1,2,3) .
\end{gathered}
$$

The object of interest is the average number of nodes that eventually (when $k \rightarrow \infty$ ) support statuses 1 and 2, $N_{1}, N_{2}, N_{3}$, $N_{j 1}$ and $N_{j 2},(j=1,2,3)$,compared to the total number of nodes $\mathrm{N}$ and $\mathrm{N}_{3}$, respectively, in the network.

In order to facilitate the mathematical analysis, we rewrite our model as

$$
\begin{aligned}
p_{i}^{1}(k+1)= & \theta(k) p_{i}^{3}(k) f_{i}+(1-\theta(k)) p_{i}^{3}(k)\left(1-g_{i}\right) f_{i} \\
& +a_{1} p_{i}^{1}(k) \\
p_{i}^{2}(k+1)= & \theta(k) p_{i}^{3}(k)\left(1-f_{i}\right) g_{i}+(1-\theta(k)) p_{i}^{3}(k) g_{i} \\
& +a_{2} p_{i}^{2}(k) \\
p_{i}^{3}(k+1)= & p_{i}^{3}(k)\left(1-f_{i}\right)\left(1-g_{i}\right)+\left(1-a_{1}\right) p_{i}^{1}(k) \\
& +\left(1-a_{2}\right) p_{i}^{2}(k)
\end{aligned}
$$

And $f_{i}, g_{i}$ and $\theta(k)$ as

$$
\begin{gathered}
f_{i}(k)=1-\prod_{j=1}^{N}\left[1-\beta a_{i j} p_{j}^{1}(k)\right] \\
g_{i}(k)=1-\prod_{j=1}^{N}\left[1-\gamma a_{i j} p_{j}^{2}(k)\right] \\
\theta(k)=\frac{s_{i}^{31}(k)}{s_{i}^{3}(k)}
\end{gathered}
$$

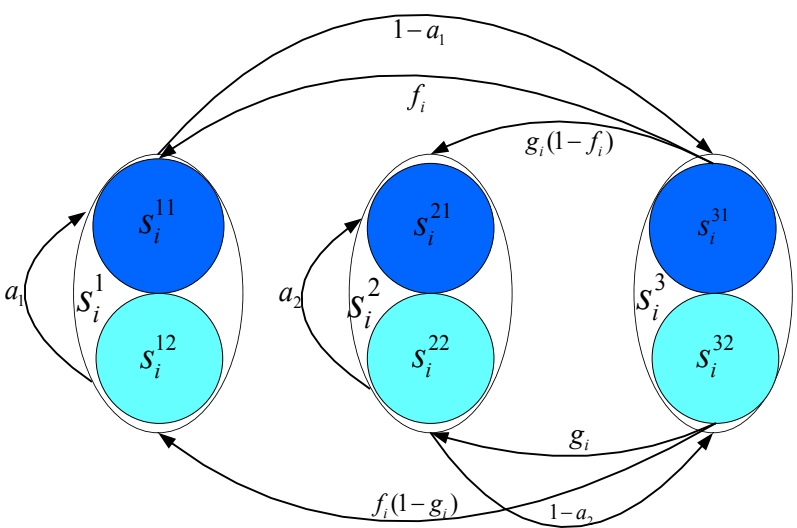

Figure 1.

The model of rumor spreading with degree-related preference mechanism.

Equivalently $N_{1}, N_{2}, N_{3}, N_{j 1}$ and $N_{j 2},(j=1,2,3)$, can be computed with Equation (3) as

$$
\begin{gathered}
N_{1}=\sum_{i=1}^{N} p_{i}^{1}(\infty), \quad N_{2}=\sum_{i=1}^{N} p_{i}^{2}(\infty), \quad N_{3}=\sum_{i=1}^{N} p_{i}^{3}(\infty), \\
N_{j 1}=\theta^{j 1}(k) \sum_{i=1}^{N} p_{i}^{j}(\infty), \\
N_{j 2}=\left(1-\theta^{j 1}(k)\right) \sum_{i=1}^{N} p_{i}^{j}(\infty),(j=1,2,3) .
\end{gathered}
$$

\section{Dynamical Systems Approach}

To simulate our model better, we adopt a dynamical systems approach to the model. We replace the probabilities for the node $i$ to support rumor 1 and 2 in Equation (4) with $x_{i}=p_{i}^{1}$ and $y_{i}=p_{i}^{2}$, respectively. The evolution of our model can be rewritten as

$$
\begin{aligned}
& x_{i}(k+1)=\left[1-x_{i}(k)-y_{i}(k)\right]\left[1-(1-\theta) g_{i}\right] f_{i}+a_{1} x_{i}^{1}(k) \\
& y_{i}(k+1)=\left[1-x_{i}(k)-y_{i}(k)\right]\left(1-\theta f_{i}\right) g_{i}+a_{2} y_{i}^{2}(k)
\end{aligned}
$$

and

$$
\begin{gathered}
f_{i}(k)=1-\prod_{j=1}^{N}\left[1-\beta a_{i j} x_{j}(k)\right] \\
g_{i}(k)=1-\prod_{j=1}^{N}\left[1-\gamma a_{i j} y_{j}^{2}(k)\right] \\
\theta(k)=\frac{s_{i}^{31}(k)}{s_{i}^{3}(k)}
\end{gathered}
$$

Equation (6) denotes a nonlinear dynamical system F: $[0,1]^{2 N} \rightarrow[0,1]^{2 N}$. Since $x_{i}$ and $y_{i}$ are probabilities, and assuming that the corresponding graph is connected, then the ergodicity of the Markov chain of the whole system is guaranteed, if $a_{1}, \beta, \gamma \neq 1, a_{2}, \beta, \gamma \neq 1$.

Therefore, when condition (8) is satisfied, dynamical system (6) has a unique globally stable fixed point. System (6) has a fixed point at $\left(x_{i}, y_{i}\right)=(0,0)$ for all $i$.

Chakrabarti and Draief etc have already been proved the ex- 
istence of a network threshold $1 / \lambda$ in several epidemiological models of virus spreading e.g. SIS model and SIR model (Chakrabarti, 2008; Draief, 2008). This threshold value is a critical point in the system dynamics, and is not related to node specific thresholds in the class of threshold models. We follow this idea, adopt the Jacobian matrix of system (6) to evaluate the local stability of the fixed point, and the result proved the threshold of our model is $1 / \lambda$.

\section{Behavior of the Model on Small-World Network}

We now investigate the model behavior for small-world network topologies. Small-world characteristics have been testified in many real world networks and social networks. We use the NW model (Newman, 1999) for generating the networks. According to the algorithm, we generate a ring lattice, where each node has $\mathrm{K}$ neighbors, $\mathrm{K} / 2$ in the clockwise, and $\mathrm{K} / 2$ in the anticlockwise direction. Each edge is added with probability $\phi$, forbidding self-loops or multiple edges between nodes. Additionally, all experiments start with one node in status 1 and one in status 2, which can change their status as time progresses. Moreover, in most of experiments rumor 1 and 2 are nearly equal, so as to observe their spread in the networks.

Figure 2 depicts the steady-state behavior of our model when the rewiring parameter $\phi$ is changed. The results are shown for networks of two different sizes with the same clustering coefficient of the initial ring lattice. The fraction of nodes in each status is given, as well as the fraction of nodes with high degree in state $j,(j=1,2,3)$, normalized by $\theta^{j 1}(k)$, respectively.

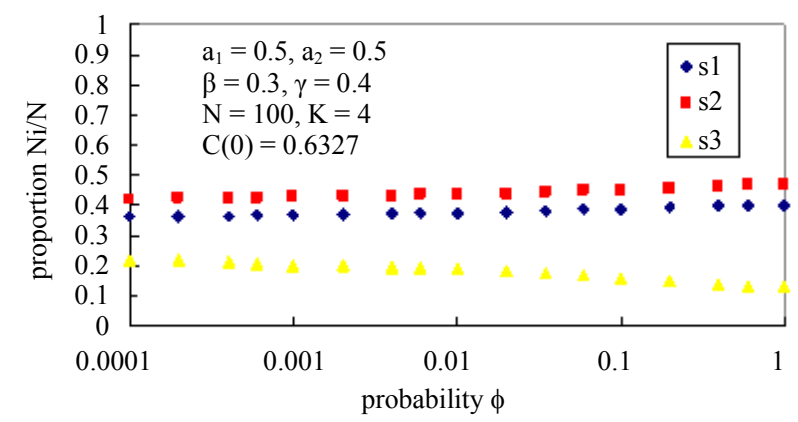

(a)

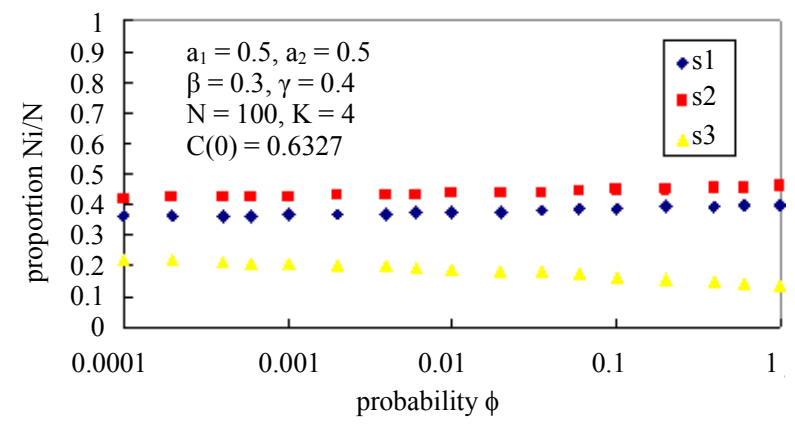

(b)

Figure 2.

The final-state behavior of the model for different values of $\phi$. The parameters include the fraction of nodes in each status and normalized clustering coefficient. (a) Results obtained from 100 nodes network realizations for each $\phi$, run for $k=500$ time units; (b) Results obtained from 1000 nodes network realizations run for $k=1000$ time units.

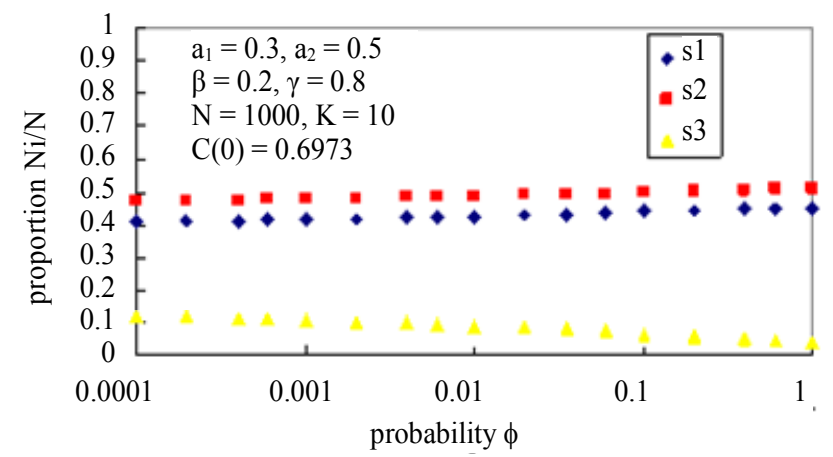

Figure 3.

The final-state behavior of the model for networks generated with the NW small-world algorithm, using a starting ring lattice with 1000 nodes and $\mathrm{K}=10$.

Evidently, the results are the same due to the same level of clustering in the networks. Furthermore, for the particular values of the model parameters, status 1 and 2 also present a fixed proportion. The cliquishness of the environment enables rumor 1 to occupy a majority of high-degree nodes. However, as the networks become increasingly random, status 2 expanded mostly at the expense of status 1 . The high degree clustering in the small-world networks undermines the spreading of the rumor 1 .

Moreover, if the level of clustering in the networks is lower, i.e., the cliquish neighborhoods are less, as in Figure 3, then status 2 is the main remaining status, even if the parameters of rumor 1 are much higher than those of rumor 2. The low clustering of the networks acts to strengthen the influence of status 2 .

In conclusion, few neighborhoods in a small-world network disable the influence of the preferred rumor 1, and strengthening the spread of rumor 2. Rumor 1 is also easy to spread through many long-range connections, quickly spreading the rumor. While, Rumor 2 could only be the main status if a small-world network does not have large highly connected groups of nodes.

\section{Conclusion and Discussion}

In conclusion, we present a model of two rumors' spreading in this paper, both of which are always preferred by a given population in a network. In this model, rumors are spreading through small-world networks, in which every node is classified into two kinds: high-degree and low-degree. And each node has one of three potential states: 1, 2 and 3, corresponding to supporter of rumor 1 or 2 and neutral. Our model follows the idea of SIS model, and can be reduced to it when some key parameters are changed. This model also has an intrinsic propagating threshold $1 / \lambda$ for rumor spreading, where $\lambda$ is the largest given value of adjacency matrix $A$, as previous studies proved. There is also a unique stable fixed point for our model, implying the choice of starting rumor 1 and 2 supporters.

We also present the preference of rumor 1 or 2 when the distribution degree of nodes is even enough and/or when the network does not contain large clustered groups of nodes. This is preformed in the model behavior on NW small-world network. The number of nodes supporting rumor 1 and 2 is well approximated, which is similar in BA networks as the critical degree $m_{0}$ is increased. 


\section{Acknowledgements}

The work described in this paper was supported by the grant (Grant No. 9024030) from the National Natural Science Foundation of China.

\section{REFERENCES}

Cane, V. R. (1966). A note on the size of epidemics and the number of people hearing a rumour. J. R. Stat. Soc. Ser. B, 28, 487-490.

Chakrabarti, D., Wang, Y., Wang, C., Leskovec, J., \& Faloutsos, C. (2008). Epidemic thresholds in real networks. ACM Transactions on Information and System Security, 10, 1-26. doi: $10.1145 / 1284680.1284681$

Christley, R. M. et al. (2005). Infection in social networks: Using network analysis to identify high-risk individuals. American Journal of Epidemiology, 162, 1024-1031. doi:10.1093/aje/kwi308

Daley, D. J., \& Kendall, D. G. (1964). Epidemics and rumours. Nature, 204, 1118. doi:10.1038/2041118a0

Daley, D. J., \& Kendall, D. G. (1965). Stochastic rumours. Journal of Applied Mathematics, 1, 42-55. doi:10.1093/imamat/1.1.42

Draief, M., Ganesh, A., \& Massoulie, L. (2008). Thresholds for virus spread on networks. Annals of Applied Probability, 18, 359-378. doi:10.1214/07-AAP470

Goldenberg, J., Libai, B., Moldovan, S., \& Muller, E. (2007). The NPV of bad news. International Journal of Research in Marking, 24, 186200. doi:10.1016/j.ijresmar.2007.02.003

Granovetter, M. (1978). Threshold models of collective behavior. American Journal of Sociology, 83, 1420-1443. doi:10.1086/226707
Guardiola, X., Diaz-Guilera, A., Perez, C. J., Arenas, A., \& Llas, M. (2002). Modeling diffusion of innovations in a social network. Physical Review E, 66, Article ID: 026121. doi:10.1103/PhysRevE.66.026121

Kempe, D., Kleinberg, J., \& Tardos, E. (2003). Maximizing the spread of influence in a social network. Proceeding of the 9th ACM SIGKDD International Conference on Knowledge Discovery and Data Mining (pp. 137-146). New York: ACM Press. doi: $10.1145 / 956750.956769$

Landahl, H. D. (1953). On the spread of information with time and distance. Bulletin of Mathematical Biology, 15, 367-381. doi:10.1007/BF02476410

Liu, Y. H. et al. (2011). Rumor riding: Anonymizing unstructured peer-to-peer systems. IEEE Transactions on Parallel and Distributed Systems, 22, 464-475. doi:10.1109/TPDS.2010.98

Newman, M. J., Watts, D. J. (1999). Renormalization group analysis of the small-world network model. Physics Letters A, 263, 341-346. doi:10.1016/S0375-9601(99)00757-4

Rapoport, A., \& Rebhun, L. I. (1952). On the mathematical theory of rumor spread. Bulletin of Mathematical Biology, 14, 375-383. doi:10.1007/BF02477853

Sudbury, A. (1985). The proportion of the population never hearing a rumour. Journal of Applied Probability, 22, 443-446. doi: $10.2307 / 3213787$

Trpevski, D. (2010). Model for rumor spreading over networks. Physical Review E, 81, Article ID: 056102. doi:10.1103/PhysRevE.81.056102

Watson, R. (1987). On the size of a rumour. Stochastic Processes and Their Applications, 1, 141-149. doi:10.1016/0304-4149(87)90010-X 